\title{
The Vela Pulsar, the Key?
}

Richard Dodson ${ }^{1}$, Dion Lewis ${ }^{2}$, David Legge ${ }^{2}$, Peter McCulloch $^{2}$, John Reynolds $^{3}$, David McConnell ${ }^{3}$, Avinash Deshpande ${ }^{4}$

${ }^{1}$ ISAS, Sagamihara, Kanagawa, Japan, 229-8510

${ }^{2}$ University of Tasmania, Hobart, Australia

${ }^{3}$ ATNF, CSIRO, Australia

${ }^{4}$ Arecibo Observatory, NAIC, USA

\begin{abstract}
Of all pulsars known, Vela has been one of the most productive in terms in understanding pulsars and their characteristics. We present the latest results derived from Australian telescopes. These include a more accurate pulsar distance, a more precise pulsar local space velocity, a new model of spin-up at a glitch, and the association of a radio nebula with the X-ray pulsar wind nebula.
\end{abstract}

\section{Introduction}

We have observed the Vela pulsar with a range of Australian telescopes. Using the Long Baseline Array (LBA) we have measured the parallax/distance of the pulsar. Using the Australia Telescope Compact Array (ATCA) we have found the radio pulsar wind nebula (PWN) that surrounds the X-ray PWN. Using the University of Tasmania's dedicated pulsar monitoring telescope in Hobart we have detected core interactions in the pulsar spin-up in the glitch of 2000 .

\section{Observations}

The pulsar monitoring telescope at Hobart is a 14-m radio telescope dedicated to timing the Vela pulsar. It collects three frequencies $(635,990,1340 \mathrm{MHz})$, and the central one is collected unfolded for high resolution timing analysis (Dodson et al. 2002). The "fast component" observed, which was fitted with a 1.2-min decay time, has been reanalyzed with a more realistic model. We marginally detect core interaction in the spin up (Lewis 2003).

The ATCA has been used to map the radio PWN at $21 \mathrm{~cm}, 13 \mathrm{~cm}, 6 \mathrm{~cm}$ and $3 \mathrm{~cm}$. Because we used compact configurations with better sensitivity to low surface brightness objects we are able to map the whole nebula, unlike previous observations (Bietenholz et al. 1991).

We have used a single baseline from the LBA to measure the sky motion of the Vela pulsar compared with the extra-Galactic source Vela-G. We have measured the proper motion and parallax of the pulsar to an unprecedented accuracy $\left(\mu_{\alpha \cos \delta}=-49.68 \pm 0.06 \operatorname{mas~yr}^{-1}, \mu_{\delta}=29.9 \pm 0.1 \operatorname{mas~yr}^{-1}, \pi=\right.$ $3.5 \pm 0.2 \mathrm{mas})$, and have been able to convert these back to the space velocity 

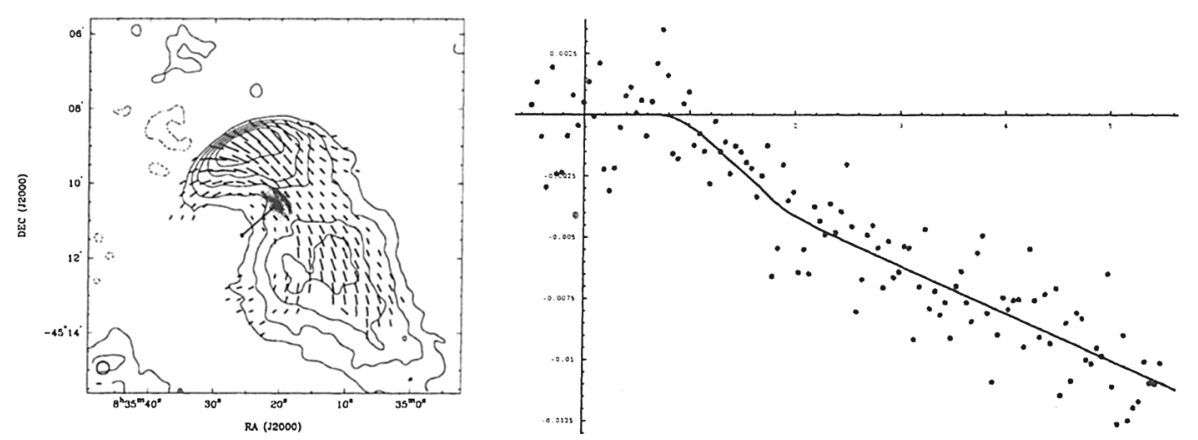

Figure 1. Left panel: The Chandra observation of the Vela PWN (grey scale) and the $5 \mathrm{GHz}$ radio contours $(-1,1,2,3,4,5 \mathrm{mJy} /$ beam). The derotated magnetic field lines are overlaid. The proper motion vector shows the distance travelled in 1000 years, and ends with the $3 \sigma$ error ellipse. Right panel: 3 -sec integrations of the single pulse data across the glitch of 2000 . The fit is for the new model of crustcore interaction, and illustrates the limits on this from the data.

and position angle of the pulsar in its local environment with greater precision that previously possible $\left(61 \pm 2 \mathrm{~km} \mathrm{~s}^{-1}\right.$ at $\left.301^{\circ} \pm 1^{\circ} 8\right)$, because of the unambiguity in the radio reference frame. Once an arithmetic error in Caraveo et al. (2001) is corrected, their results agree with ours (Caraveo, personal communication).

\section{Future Observations}

A coherently dedispersed $30 \mathrm{MHz}$-bandwidth backend at $635 \mathrm{MHz}$ on the pulsar timing telescope should allow an increase in sensitivity of an order of magnitude over previous observations. We will also observe the radio nebula at the ATCA at higher frequencies to find the turn-over frequency and model the emission from the X-rays down to the radio frequencies, and we are observing at the VLA to get sensitive rapid observations in order to measure changes associated with the recently discovered X-ray outer jet (Pavlov et al. 2001). Finally, the limitation in the accuracy of the VLBI observation is the solar motion parameters, and we cannot improve on this. Nevertheless we are planning to use Vela as a demonstration source in a baseband e-VLBI experiment.

\section{References}

Bietenholz, M. F., Frail, D. A., \& Hankins, T. H. 1991, ApJ, 376, L41

Caraveo, P. A., De Luca, A., Mignani, R. P., \& Bignami, G. F. 2001, ApJ, 561, 930

Dodson, R. G., McCulloch, P. M., \& Lewis, D. R. 2002, ApJ, 564, L85

Lewis, D. 2003, PhD thesis, University of Tasmania

Pavlov, G. G., Kargaltsev, O. Y., Sanwal, D., \& Garmire, G. P. 2001, ApJ, 554, L189 